\title{
Research on De-noising of Fault Signal Based on Improved Multi-wavelets
}

\author{
ZHANG Jiali ${ }^{1}$, WANG Jie ${ }^{1}$, WANG Wenfeng ${ }^{1,2,3}$, ZHANG Lin ${ }^{1, a}$, ZHAO Haizhou ${ }^{1}$, LEI Rongqiang ${ }^{1}$ \\ ${ }^{1}$ Air Defense and Antimissile Institute, Air Force Engineering University, Xi'an,710051, China \\ 2. The 302ed Institute of the Tenth Academy of China Aerospace Science \& Industry Corporation; Guiyang 550009, \\ China \\ ${ }^{3 .}$ School of Automation Science and Electrical Engineering, Beihang University, Beijing 100191, China
}

\begin{abstract}
Aiming at signal de-noising of fault diagnosis, we need obtain fault signal of high signal to noise ratio with multi-wavelets which need little prior knowledge and are uninfluenced by waveform matching. Here, this paper determines the wavelet basis through contrast experiment, and the decomposition level is confirmed by the principles of making noise fraction from details and making low energy from general picture, threshold is ensured by the method of midpoint optimization. The simulation shows that this method can enhance signal to noise ratio obviously.
\end{abstract}

\section{Introduction}

Fault diagnosis is to find out the reasons of object's triggering some operation function being not consistent with normal condition in the case of given working environment, judge the elements of arousing the occurrence of this condition, and even forecast the tendency and the loss of the development of the condition[1-5]. Basic flow path can be divided into four procedures commonly as Figure 1 shows:

1. Signal collection. Collect the representative fault signal in different conditions.

2. Signal de-noising. Dislodge the interfering signal in the collection signal.

3. Feature extraction. Extract the signal of reflecting fault's feature in the time-domain, frequency-domain or time- frequency domain of de-noising signal.

4. Fault diagnosis. According to relevant algorithm, judge the fault of characteristic signal.

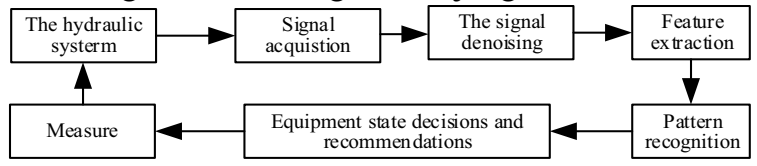

Figure 1.The flow chart of fault diagnosis.

As the key of fault diagnosis, fault de-noising normally has some methods at present, such as repeating average de-noising, multipoint smooth de-noising, fourier de-noising, wavelet de-noising

\footnotetext{
${ }^{a}$ Corresponding author : dreamland_0628@163.com
} 
and so on [6-9]. As a new method of signal de-noising in recent years, wavelet transform is a method of time-frequency analysis [10]. It can analyze a signal's details, and at the same time, can see the whole outline of signal through transforming time and frequency partly.

The scale function of multi-wavelet is generated by two or more functions, so it is less affected by wave form matching and has little requirement on the signal's prior knowledge. What's more, it can combine the smooth, symmetry, tight branch, perpendicularity and vanishing moment well. This paper obtains fault signal of high signal to noise ratio through improving multi-wavelet determination method in wavelet basis, wavelet decomposition level and various detail thresholds.

\section{Improving Multi-wavelet Algorithm}

\subsection{The Brief Introduction of Multi-wavelet}

When conducting signal de-noising process, multi-wavelet follows the steps as Figure 2 shows. Firstly, pre-filter noise signal. Secondly, perform threshold to fault signal that has experienced multi-wavelet decomposition. Thirdly, obtain the new reconfiguration signal through multi-wavelet inverse transformation. Finally, achieve target value by using post-filtering to dispose fault signal.

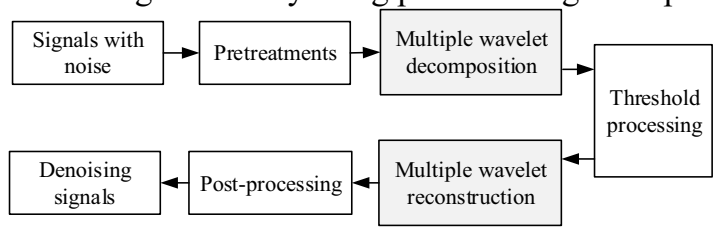

Figure 2. The process of wavelet de-noising.

The multi-resolution analysis of multi-wavelet de-noising consists of multi-scale function, but $L^{2}(R)$ space base consists of many traditional and flexible wavelet functions. Suppose $\Phi(t)=\left[\varphi_{1}(t), \varphi_{2}(t), \cdots, \varphi_{r}(t)\right]$ as $r$ dimension scale function, $\Psi(t)=\left[\psi_{1}(t), \psi_{2}(t), \cdots, \psi_{r}(t)\right]$ as wavelet function, $h_{k}$ and $g_{k}$ as $r \times r$ dimension filter array. Therefore, the equation is

$$
\begin{array}{ll}
\Phi(t)=\sum_{k} h_{k} \Phi(2 t-k) & k \in Z \\
\Psi(t) & =\sum_{k} g_{k} \Phi(2 t-k) \quad k \in Z
\end{array}
$$

Set $s_{j-1, n}$ as $r$ dimension low-frequency component, $d_{j-1, n}$ as $r$ dimension high-frequency component, $h_{k}^{*}, g_{k}^{*}$ as the conjugate transpose of $h_{k}$ and $g_{k}$. The decomposition and reconfiguration of multi-wavelet follow the equation

$$
\begin{aligned}
& s_{j-1, n}=\sum_{k} h_{k-2 n} s_{j, k}, d_{j-1, n}=\sum_{k} g_{k-2 n} s_{j, k} \\
& s_{j, n}=\sum_{n}\left(h_{k-2 n}^{*} s_{j-1, n}+g_{k+2 n}^{*} d_{j-1, n}\right)
\end{aligned}
$$

\subsection{The Choice of Multi-wavelet Index}

In the procedure of multi-wavelet signal de-noising, three prime factors of affecting the effect of signal de-noising are the structure of multi-wavelet, the number of piles choice of wavelet decomposition, and the determination of detail threshold.

\subsubsection{The structure of multi-wavelet}

Wavelet transformation is different from Laplace transform and Fourier transform that have only one fixed kernel function. Not only does it not have fixed wavelet base function, it also has great difference in systematic ness, support length and wave path .However, the three conditions as follows must be fitted for wavelet base function: 
Allowance condition: $\int_{-\infty}^{+\infty} \frac{|\omega|^{2}}{\omega} d \omega<\infty$ which guarantees the inversion geometry of wavelet transform. And volatility: $\int_{-\infty}^{+\infty} \psi(t) d \omega=0$.

Time-frequency attenuating property: the frequency spectrum of wavelet base function must be committed in a relevant narrow frequency ban. And at the same time, in time threshold, it also must be concentrated on stated small interval.

Besides, when choosing wavelet base function for the practical apply, it is necessary to consider perpendicularity, symmetry, tight branch, vanishing moment and similarity synthetically.

In addition to the conditions above, the choice of multi-wavelet base should follow the principles of high time-frequency resolution ratio, small decomposition index ratio and restructure error, estimated variance approaching practice. Among the three conditions, the prior two conditions are used to improve the effect of signal de-noising and the third condition is used to simplify calculating steps.GHM multi-wavelet is symmetrical, double orthogonal multi-wavelet, obtained by Geronimo Hardin and Massopust through complicated and fractal interpolation method. Commonly, we use GHM multi-wavelet and Db2 wavelet to process the simulation signal of the pitting fault of bearing outer ring, and prove the superiority of GHM in the aspect of signal de-noising .Therefore, this paper choose GHM to conduct de-noising.

\subsubsection{The choice of number plies of multi-wavelet decomposition}

Set the number of plies of multi-wavelet decomposition of collection frequency as $f_{s}$, the maximal frequency of the prime energy interval of fault signal as $f_{m}\left(f_{m}>0\right)$.if prime energy is in the frequency scale of the latest plié of general picture, then

$$
\frac{f_{s}}{2^{n+1}}>f_{m}
$$

Signal experienced multi-wavelet decomposition at least exists a detail component $(n \geq 1)$, so that we can obtain equation (5), what's more, we obtain equation(6)

$$
\begin{aligned}
& \frac{f_{s}}{2^{n+1}} \leq \frac{f_{s}}{2^{2}} \\
& f_{s}>2^{2} f_{m}
\end{aligned}
$$

The more the number of plies of wavelet is, the more detail component will be generated and the more amount of noise will be wiped out. We can obtain the most detail components based on equation (7).Because the number of plies of multi-wavelet decomposition is a non-negative positive integer, the final certain equation of the greatest number of plies of wavelet decomposition of wavelet de-noising can be indicated as equation (8).Literature has detailed discussion.

$$
\begin{gathered}
f_{s} /_{2^{n+2} \leq f_{m} \leq} f_{s} /_{2^{n+1}} \\
n=\operatorname{ent}\left(\mathrm{lb} \frac{f_{s}}{2^{2} f_{m}}\right)
\end{gathered}
$$

\subsubsection{The determination of various detail thresholds of multi-wavelet}

In the common methods of wavelet threshold processing, necessary characteristic component energy only fixes on some limited wavelet coefficient, however, the noise energy of fault signal almost scatters to the whole wavelet domain, so it needs to choose appropriate threshold . Set $\lambda=\sigma \sqrt{2 \ln N}$ ( $N$ is the length of signal), $\sigma$ as the standard deviation of noise. It is regular to use equation (9) to estimate threshold in engineering.

$$
\sigma=\frac{\text { median }\left|w_{i, j}\right|}{0.6745}
$$

So the definition of hard threshold method is: 


$$
\widehat{w}_{i, j}=\left\{\begin{array}{cc}
w_{j, k}, & \left|w_{j, k}\right| \geq \lambda \\
0 & \left|w_{j, k}\right|<\lambda
\end{array}\right.
$$

In hard threshold function, wavelet coefficient $\widehat{w}_{i, j}$ is in continuous between $\lambda \lambda$ and $-\lambda$.

$$
\widehat{w}_{i, j}=\left\{\begin{array}{cc}
\operatorname{sgn}\left(w_{j, k}\right)\left(\left|w_{j, k}\right|-\lambda\right), & \left|w_{j, k}\right| \geq \lambda \\
0 & \left|w_{j, k}\right|<\lambda
\end{array}\right.
$$

In soft threshold function, because of the contract processing of wavelet coefficient, there is unavoidable error between $\widehat{w}_{i, j}$ and $w_{j, k}$. Meanwhile, it is not consistent, which generates error hard to be eliminated in hard threshold method and soft threshold method. In order to make up for the inconsistence of hard threshold method and reduce the constant error of soft threshold method, this paper uses a new threshold function, the function is as equation (12) shows:

$$
\widehat{w}_{i, j}=\left\{\begin{array}{cc}
\operatorname{sgn}\left(w_{j, k}\right)\left(\left|w_{j, k}\right|-\alpha \lambda-\frac{\lambda-\alpha \lambda}{\exp \left(w_{j, k}-\lambda\right)}\right), & \left|w_{j, k}\right| \geq \lambda, 0 \leq \alpha \leq 1 \\
0 & \left|w_{j, k}\right|<\lambda
\end{array}\right.
$$

In the equation, $\alpha$ is between 0 and 1 .above equation, if $\alpha=0$, threshold curve will become soft threshold curve; if $\alpha=1$, the asymptotic line of threshold curve is hard threshold curve. Although, using the strike an average method, in which set $\alpha=0.5$, increases signal to noise ratio, $\alpha$ is not most one. This paper search optimal $\alpha$ (set $\alpha$ as $\left.\alpha_{\text {mid }}\right)$ by the means of mid-point method, and the flow path of the algorithm is as Figure 3 shows.

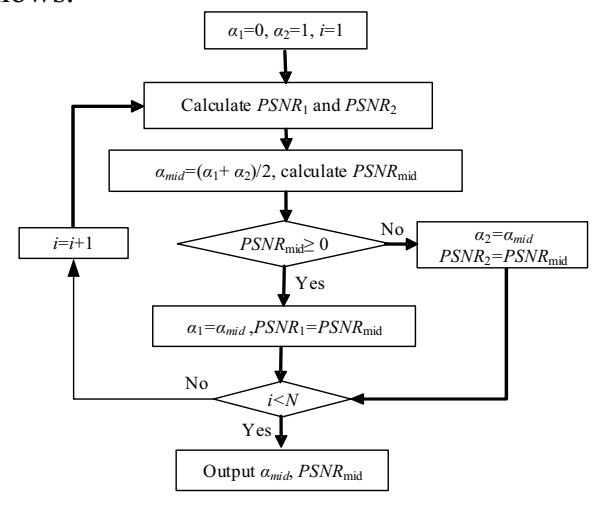

Figure 3. The algorithm calculate flow

\section{Simulation Confirmation}

In order to check the effectiveness of improving multi-wavelet algorithm, This paper chooses simulation signal $f(t)=\sin (10 \pi t)+0.7 \sin (24 \pi t)$ to check it. After adding $3 \mathrm{~dB}$ signal to noise ratio, collection frequency is set to be $1024 \mathrm{~Hz}$, and the time of lasting collection is $1 \mathrm{~s}$. And we use the method in chapter 2.2 to determine Multi-wavelet base, decomposition and threshold.

\subsection{The pretreatment and reconfiguration of GHM multi-wavelet}

As GHM is a multi-wavelet, where $r=2$, for one-dimensional fault signal $x[n]$,we can process it and obtain two-dimensional input signal $s_{0, n}$. In order to keep the trait of system first-order vanishing moment trait of system, we usually choose $c=\sqrt{2}$.

$$
s_{0, n}=\left(\begin{array}{c}
x[n] \\
c \cdot x[n]
\end{array}\right)
$$

The coefficient of low-pass of GHM multi-wavelet can be indicated as 


$$
h_{0}=\left[\begin{array}{cc}
\frac{3}{10} & \frac{2 \sqrt{2}}{5} \\
-\frac{\sqrt{2}}{40} & -\frac{3}{20}
\end{array}\right] h_{1}=\left[\begin{array}{cc}
0 & 0 \\
-\frac{9 \sqrt{2}}{40} & -\frac{3}{20}
\end{array}\right] h_{1}=\left[\begin{array}{cc}
0 & 0 \\
-\frac{9 \sqrt{2}}{40} & -\frac{3}{20}
\end{array}\right] h_{1}=\left[\begin{array}{cc}
0 & 0 \\
-\frac{\sqrt{2}}{40} & 0
\end{array}\right]
$$

The coefficient of high-pass of CHM multi-wavelet can be described as

$$
g_{0}=\left[\begin{array}{cc}
-\frac{\sqrt{2}}{40} & -\frac{3}{20} \\
-\frac{1}{20} & -\frac{3 \sqrt{2}}{20}
\end{array}\right] g_{1}=\left[\begin{array}{cc}
\frac{9 \sqrt{2}}{40} & -\frac{1}{2} \\
\frac{9}{20} & 0
\end{array}\right] h_{1}=\left[\begin{array}{cc}
\frac{9 \sqrt{2}}{40} & -\frac{3}{20} \\
-\frac{9}{20} & \frac{3 \sqrt{2}}{20}
\end{array}\right] h_{1}=\left[\begin{array}{cc}
-\frac{\sqrt{2}}{40} & 0 \\
\frac{1}{20} & 0
\end{array}\right]
$$

When retreating GHM multi-wavelet, we can retreat it longitudinally, and retreat it breadthwise, then process post-filtering .Post-filter $Q(k)(k=0,1)$ can be indicated as

$$
Q_{0}=\left[\begin{array}{cc}
0 & 0 \\
\frac{4 \sqrt{2}}{5} & -\frac{3}{10}
\end{array}\right] Q_{1}=\left[\begin{array}{cc}
0 & 0 \\
0 & -\frac{3}{10}
\end{array}\right]
$$

\subsection{Simulation Experiment}

Simulation original signal and noise signal are as Figure 4 shows. Bring $f_{s}=1024$ and $f_{m}=12$ into equation (8), and obtain the optimal number of plies of plies of multi-wavelet decomposition, 4 and $\alpha$, the value of threshold function is 0.6213 based on Figure 3 .In order to judge the veracity of the number of plies of wavelet decomposition in this paper, set the number of plies of wavelet decomposition as, $n=3, n=4$ and $n=5$, under the same condition, and compare their effects of signal de-noising. The effect of simulation signal effect is as Figure 5 shows, we can see when $n=3$, the effect wave form of signal de-noising is obviously variant, and when $n=4$ or $n=5$, the effect is similar.
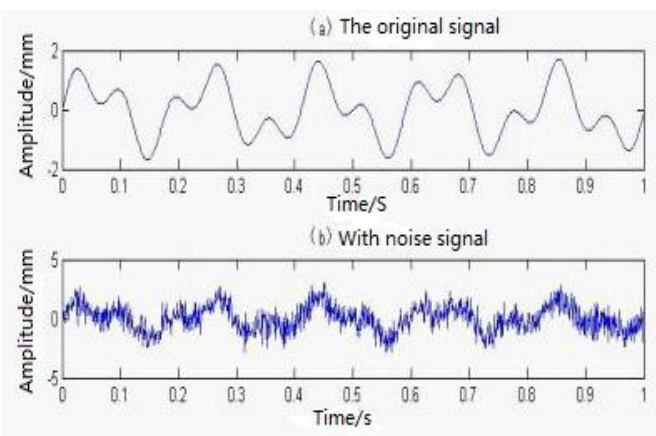
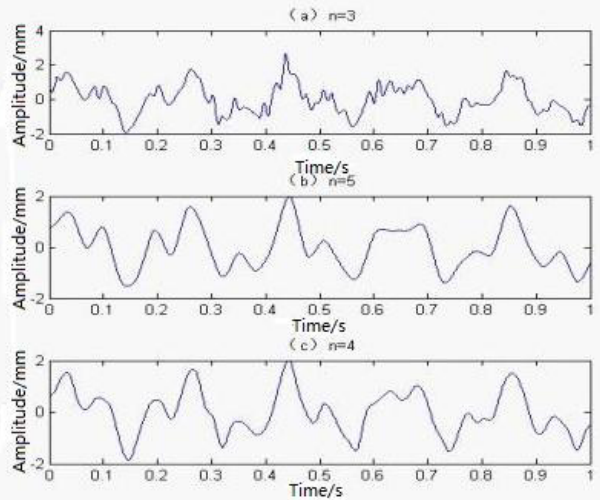

Figure 4. The simulation signal.

Figure 5. The simulation signal de-noising effect.

Figure 6 provides the de-noising effect frequency spectrum of simulation signal, we can see that when $n=5$, frequency component changes greatly besides $20 \mathrm{~Hz}$, which is different from original signal. When $n=3$, de-noising signal exists error in high frequency. However, when $n=5$, the prime frequency trait of simulation signal is the same as original signal. Therefore, we can draw a conclusion that when $n=4$ the effect is best. To sum up, the proposed method of wavelet decomposition can resolve low frequency into general picture effectively.

In order to check the effectiveness of detail threshold determination method proposed in this paper, the effects of hard threshold de-noising, soft threshold de-noising and threshold de-noising given by this paper are as Figure 7 shows in the same condition, the effect of threshold de-noising proposed in this paper is obviously better than the effect of soft threshold de-noising and hard threshold de-noising. 

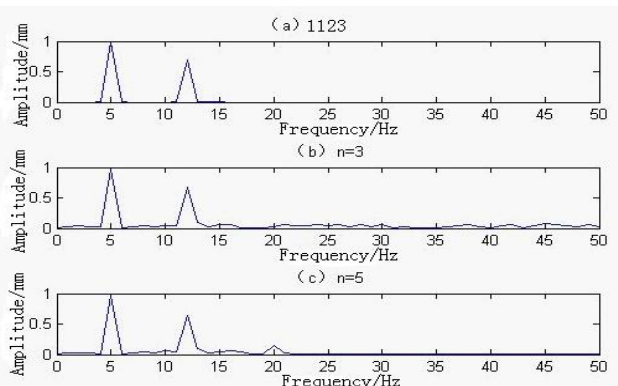

(d) $n=4$

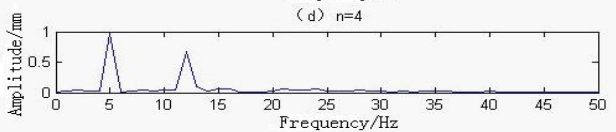

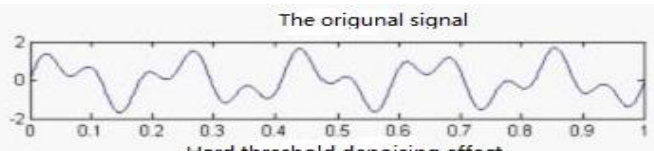
Hard threshold denoising effect

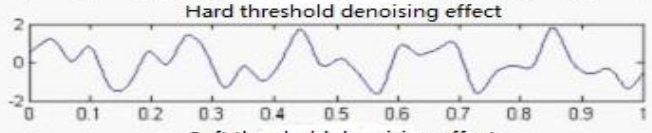

Soft threshold denoising effect

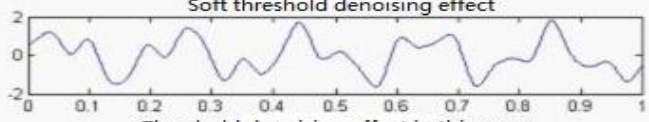

Threshold denoising effect in this paper

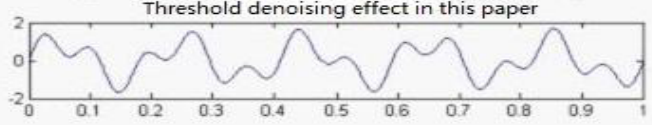

Figure 6. The signal de-noising effects of frequency domain. Figure 7. De-noising effect with different thresholds.

\section{Conclusion}

According to the principles of reflecting the low-frequency component decomposed into general picture and decomposing noise component into details, we choose the method of the number of plies of wavelet decomposition in fault signal de-noising. Through a midpoint optimization method, we determine the optimal in wavelet de-noising threshold parameter, ensuring the fault signal with high signal-to-noise ratio. Meanwhile, simulation experiments have verified the correctness of this method.

\section{References}

1. Wu Jinpei, Xiao Jianhua. Intelligent fault diagnosis and expert system. Science Press.(1997)

2. Wang Yilong, Yang Shouzhi. The wavelet denoising method of based consistent threshold function. Shan Tou University Academic Journal (Natural Science), 28(4):66-74.(2013)

3. Mallat S,Hwang W L. Singularity detection and processing with wavelet. IEEE Transaction Information Theory, 38(2):617-643.(1992)

4. Lavrik I, Young Jung Y, Ruggeri F, Vidakovic B. Bayesian false discovery rate wavelet shrinkage: theory and applications. Communications in Statistics-Simulation and Computation, 37(6): 1086-1100.(2008)

5. Xu, Y., Weaver, J. B., Healy, Jr., D. M., etal. Wavelet transform domain filters a spatially selective noise filtration technique. IEEE Trans Image Processing, 3(6): 747-758.(1994)

6. Banham M R, Katsaggelos A K. Spatially adaptive wavelet-based multiscale image restoration. IEEE Trans. Image Processing, 5(4): 619-634.(1996)

7. Mariantonia Cotronei, Laura B Mntefusco, Luigia Puccio. Multiwavelet analysis and signal processing. IEEE Transactions on Circuits and Systems- II : Analog and Digital Signal Processing, 45(8): 970-987.(1998)

8. Gao Taichang, Han Xiaodong, Qu Shi. The electric field variability signal denoising of multi-wavelet transform. The PLA university of science and technology Academic Journal, 10(12):90-94.(2009)

9. Xiang Dongyang, Wu Zhengguo, Hou Xinguo, Zhao Hua. Improving the denosing algorithm of multi-wavelet transform coefficient. High voltage technology, 37(7):1728-1733.(2011)

10. Zhang Wenjuan, Zhou Dandan,Wang Ling. A improvement project of wavelet threshold denoising. Computer Development and Applying, 20(9):28-29.(2007) 